\title{
The SIRT 3 Expression Profile is Associated with Pathological and Clinical Outcomes in Human Breast Cancer Patients
}

\author{
Shaozhong He ${ }^{a, b}$ Chun He ${ }^{b}$ Hua Yuan ${ }^{a}$ Shizhong Xiong ${ }^{b}$ Zhenyu Xiao ${ }^{b}$ Longhua Chen $^{a}$ \\ aDepartment of Radiation Oncology, Nanfang Hospital, Southern Medical University, Guangzhou, \\ bDepartment of Oncology, the People's Hospital of Ganzhou, Ganzhou, China
}

\section{Key Words}

SIRT 3 - Breast cancer • Clinical characteristics $•$ Prognosis

\begin{abstract}
Aims: To investigate the association of Sirtuin 3 (SIRT 3) expression between the clinical characteristics and prognosis in breast cancer patients. Methods: 308 female patients with histologically confirmed breast cancer were enrolled in this study. The SIRT 3 expressions in tumor samples were detected. All the patients were followed up overall survival time (OS) and disease-free survival (DFS) time. Results: SIRT 3 expression was significantly correlated with clinical characteristics including lymph node metastasis, pathological grade and tumor size of breast cancer. SIRT 3 expression status also affected the DFS and OS of breast cancer. Patients with high expression of SIRT 3 had shorter DFS and OS than those with low expression. Univariate and multivariate Cox analyses confirmed that high SIRT 3 expression predicted a poor prognosis in breast cancer patient. In vitro study revealed that the SIRT 3 knockdown by small interfering RNA technique dramatically reduced the proliferation, migration and invasion of breast cancer cell lines. Conclusion: Our results suggest that SIRT 3 may serve as a marker for clinical feature and prognosis for breast cancer.
\end{abstract}

Copyright $(2014$ S. Karger AG, Basel

\section{Introduction}

Breast cancer is the most common malignancy in women and is the second leading cause of cancer deaths in women [1,2]. Although the incidence of breast cancer is lower in China compared to that in western countries, it has increased by $80 \%$ in young women 
in the past two decades, with a total increase in 50-100 \% [3-5]. The prognosis for breast cancer has been improved in recent decades, however, the clinical outcome of patients with metastatic breast cancer remains poor, with a median overall survival period of 2 to 3 years [6-10].

Sirtuin 3 (SIRT 3) is a member of Sirtuin family, the mammalian homologues of the silent information regulator 2 first discovered in Saccharomyces cerevisiae as an NAD+-dependent histone deacetylase $[11,12]$. The other Surtuin members, especially SIRT1 has been reported to be associated with the clinical features and prognosis of breast cancer. e.g., inhibition of SIRT1 by small molecule inhibitors reduces the levels of aromatase mRNA in breast cancer cells and SIRT1 is significantly up-regulated in invasive breast ductal carcinoma [13]. SIRT1 and SIRT2 positively regulate the levels of Rac1-GTP and the activity of T-cell lymphoma invasion and metastasis [14]. SIRT1 regulates dishevelled proteins and promotes transient and constitutive Wnt signaling in several cancer cell lines including breast cancer cells [15]. SIRT1 represses estrogen-signaling, ligand-independent estrogen $\alpha$-mediated transcription and cell proliferation in estrogen-responsive breast cells [16]. SIRT1 is essential for oncogenic signaling by estrogen/estrogen receptor $\alpha$ in breast cancer [17]. However, the role of SIRT 3 in breast cancer remains largely unknown. This study was to investigate the association of SIRT 3 expression with the clinical characteristics and prognosis of breast cancer patients.

\section{Materials and Methods}

\section{Patient enrollment and Follow-up}

Three hundred and eight female patients who were histologically confirmed breast cancer and underwent radical operations in the Department of Oncology of Ganzhou People Hospital and Department of Radiation Oncology of Nanfang Hospital were enrolled in this study between January 2003 and January 2006. The inclusion criteria were as follows: (a) curative operations were performed; (b) resected specimens were pathologically examined; (c) more than 10 lymph nodes were pathologically examined after operation. All the patients included in present study did not receive any chemotherapy and radiation therapy before enrollment, and their complete clinical data, including age, menopausal status, histological type, lymph nodes status, tumor size, grade, estrogen-receptor (ER) status, progesterone receptor (PR) status, and human epidermal growth factor receptor 2 (HER2) status, were obtained and reviewed. The present study conformed to the ethical standards of the World Medical Association Declaration of Helsinki and was approved by the Ethics Committee of our hospital (2003-No. 14-11).

The follow-up results were available for all patients. Outcome data include survival status, overall survival (OS) time and disease-free survival (DFS) time. DFS and OS times were defined as the time interval from the date of surgery to the date of first recurrence or death, which were the two assessments used for prognostic analyses.

Immunohistochemical staining analysis and evaluation

Specimens were obtained immediately after excision during surgery and stored at $-80^{\circ} \mathrm{C}$ until use. The tissues were fixed, paraffin embedded, and cut to 5 - $\mu$ m-thick sections for immunohistochemistry. Briefly, the slides were deparaffinized in xylene, rehydrated in a gradient of ethanol solutions, and then pretreated in a microwave oven. Then, the slides were incubated in a humid chamber at $4{ }^{\circ} \mathrm{C}$ overnight with SIRT 3 primary antibody (1:200, sc-99143, Santa Cruz Biotechnology, Santa Cruz, CA). SIRT 3 expression were classified semiquantitatively by immunoreactive score (IRS) according to the method described previously [18]. SIRT 3 staining was defined as low expression (IRS: 0-5) and high expression (IRS: 6-12).

Tumor Cell line Culture and SiRNA transfection

Two human breast cancer cell lines MDA-MB-231 and BT-549 were cultured to 75\% confluence before small interfering RNA (siRNA) transfection. Nonspecific control siRNA or SIRT-3 siRNA (SIRT-3 siRNA sequence: CAT CCC TAC ATG CAG ATG AAA) was transfected by siLentFect Lipid Reagent (Bio-Rad, Hercules, CA, USA) for 24 hours according to the manufacturer's instructions. 


\section{Real-time RT-PCR assay}

All reagents including specific and control primers for TaqMan real-time RT-PCR were purchased from Applied Biosystems and the reactions were set according to the manufacturer's protocol. Briefly, total RNA was purified by TRIzol (Life Technologies/Invitrogen). $10 \mathrm{ng}$ of total RNA were used for reverse transcription with TaqMan MicroRNA Reverse Transcription Kit. Real-time PCR quantification was performed using TaqMan PCR primers and TaqMan Universal Fast PCR system (Applied Biosystems). The relative gene expression was determined by normalizing to reference gene glyceraldehyde phosphate dehydrogenase (GAPDH) using the relative quantitative $2-\Delta \Delta \mathrm{Ct}$ method.

\section{Western Blot Analysis}

After siRNA transfection, the cells were lysed for Western blot assay to determine SIRT 3 expression. Extracts were resolved on SDS-polyacrylamide gels followed by transfer to nitrocellulose membranes. Proteins were resolved by electrophoresis on $8-12 \%$ sodium dodecyl sulfate-polyacrylamide gels and transferred by electroblotting to polyvinylidene difluoride membranes in transfer buffer ( $25 \mathrm{mM}$ Tris$\mathrm{HCl}$ (pH 7.6), $192 \mathrm{mM}$ glycine, 20\% methanol, 0.03\% sodium dodecyl sulfate). After immunoblot analysis, membranes were immunoblotted with SIRT 3 antibody (1:1,000, sc-99143, Santa Cruz Biotechnology, Santa Cruz, CA) and glyceraldehyde-3-phosphate dehydrogenase (GAPDH, 1:1,000, Santa Cruz Biotechnology, Santa Cruz, CA). Membranes were then washed and incubated with a secondary antibody coupled to horseradish peroxidase. Immunolabeling was detected using the ECL Reagent (Amersham Biosciences).

\section{Cell Proliferation Assay}

MTT assay was performed according to manufacturer instructions (Sigma Aldrich). Briefly, cells were washed. MTT reagent (3-(4, 5-dimethylthiazol-2-yl)-2, 5-diphenyltetrazolium bromide, M 2128, Sigma Aldrich) was added and incubated at $37^{\circ} \mathrm{C}$ for $3 \mathrm{hrs}$. After MTT reagent removal, MTT solvent $(10 \%$ Triton $100 \mathrm{X}$ and $0.1 \mathrm{~N}$ HCL in anhydrous isopropanol) was added. Absorbance was determined in a Microwell plate reader (Model 680, Biorad, Marnes-La-Coquette, France).

\section{Cell Migration Assay}

After SiRNA transfection, cell migration was determined by using a modified two-chamber migration assay with a pore size of $8 \mu \mathrm{m}$. For migration assay, $1 \times 105$ cells were seeded on the upper compartment of 24-well Transwell culture chamber and $600 \mu \mathrm{l}$ of complete medium was added to the lower compartment. After $24 \mathrm{~h}$ incubation, cells were fixed with methanol. Non-traversed cells were removed from the upper surface of the filter carefully with a cotton swab. Traversed cells on the lower side of the filter were stained with crystal violet and counted.

\section{Cell Invasion Assay}

The invasion assay was performed using a modified two-chamber plates. $30 \mu \mathrm{l} \mathrm{of} 50 \mathrm{mg} / \mathrm{ml} \mathrm{Matrigel}$ (BD Biosciences, Mississauga, Canada) in serum-free medium was added to the upper compartment of 24well Transwell culture chamber. $1 \times 105$ cells suspended in $200 \mu \mathrm{l}$ of serum-free medium were seeded on the upper compartment, and $600 \mu \mathrm{l}$ of complete medium was added to the lower compartment. After $24 \mathrm{~h}$ incubation at $37^{\circ} \mathrm{C}$, cells were fixed with methanol. Non-invaded cells were removed from the upper surface of the filter carefully with a cotton swab. Invaded cells on the lower side of the filter were stained with crystal violet and counted.

\section{Statistical analysis}

All data were analyzed with SPSS statistics software (version 16.0, Chicago, IL, USA). Relationships between SIRT 3 expression status and clinical parameters were studied using chi-square test, Fisher's exact test, or independent $t$ tests. Survival was analyzed using the Kaplan-Meier method. The log-rank test was used to analyze survival differences. Univariate and multivariate analyses were performed using the Cox proportional hazards model selected in forward stepwise. A P value of $<0.05$ was considered statistically significant. 


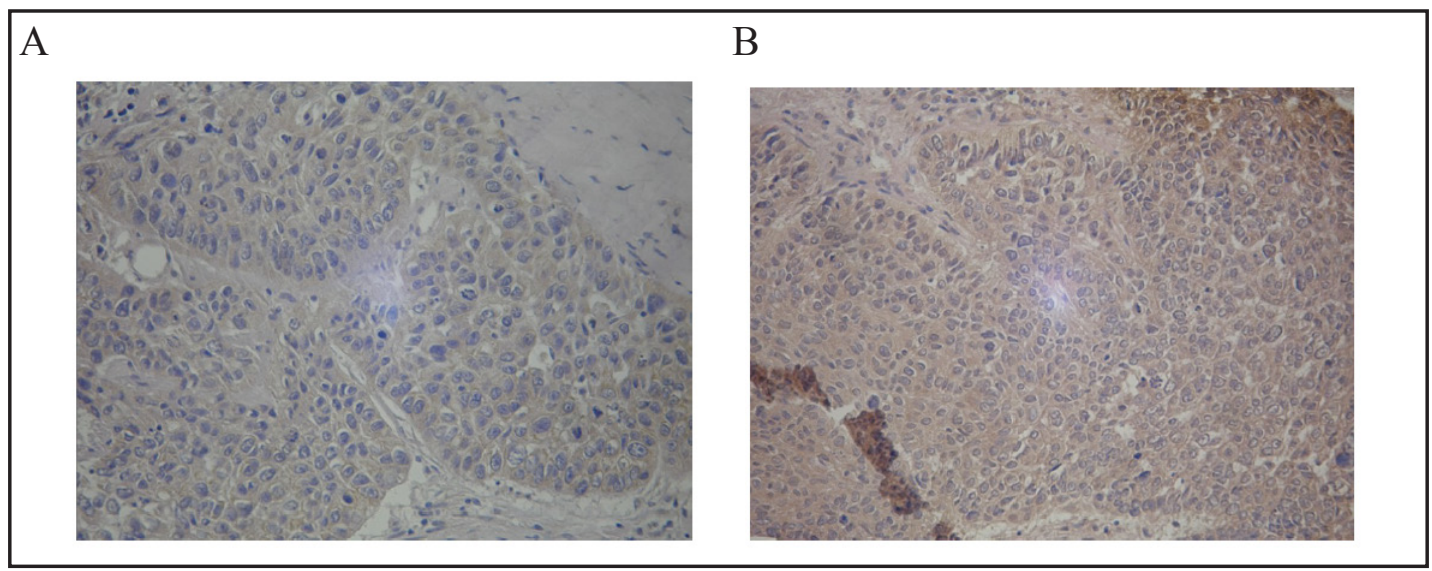

Fig. 1. The SIRT 3 protein by immunochemical staining tests was located in the nucleus in the breast tumor tissues $(\times 40)$. (a) week expression of SIRT 3. (b). high expression of SIRT 3. The immunoreactivities were primarily observed in cytoplasm. The micrographs showed low (1a), and high (1b) expression of SIRT3 in cancer tissues.

Table 1. The associations between SIRT 3 expression status and clinicopathological characteristics of breast cancer patients. ER,estrogen-receptor; $\mathrm{PR}$, progesterone receptor ;HER2, human epidermal growth factor re-

\begin{tabular}{|c|c|c|c|c|c|}
\hline & $\begin{array}{c}\text { Low } \\
\text { Expression }(n=148)\end{array}$ & & $\begin{array}{c}\text { High expression } \\
(n=160)\end{array}$ & & $P$ \\
\hline \multicolumn{6}{|l|}{ Age (years) } \\
\hline$\leq 50$ & 80 & $54.05 \%$ & 82 & $51.25 \%$ & 0.353 \\
\hline$>50$ & 68 & $45.95 \%$ & 78 & $48.75 \%$ & \\
\hline \multicolumn{6}{|l|}{ Menopausal status } \\
\hline Premenopausal & 67 & $45.27 \%$ & 79 & $49.38 \%$ & 0.272 \\
\hline Postmenopausal & 81 & $54.73 \%$ & 81 & $50.63 \%$ & \\
\hline \multicolumn{6}{|l|}{ Histological subtype } \\
\hline Ductal & 91 & $61.49 \%$ & 101 & $63.13 \%$ & 0.429 \\
\hline Lobular & 57 & $38.51 \%$ & 59 & $36.88 \%$ & \\
\hline \multicolumn{6}{|l|}{ lymph node metastasis } \\
\hline Negative & 110 & $74.32 \%$ & 84 & $52.50 \%$ & $<0.001$ \\
\hline Positive & 38 & $25.68 \%$ & 76 & $47.50 \%$ & \\
\hline \multicolumn{6}{|l|}{ Tumor size } \\
\hline$\leq 2 \mathrm{~cm}$ & 76 & $51.35 \%$ & 46 & $28.75 \%$ & $<0.001$ \\
\hline$>2 \mathrm{~cm}$ & 72 & $48.65 \%$ & 114 & $71.25 \%$ & \\
\hline \multicolumn{6}{|l|}{ Tumor Grade } \\
\hline I, II & 108 & $72.97 \%$ & 70 & $43.75 \%$ & 0.001 \\
\hline III & 40 & $27.03 \%$ & 90 & $56.25 \%$ & \\
\hline \multicolumn{6}{|l|}{ ER status } \\
\hline Negative & 89 & $60.14 \%$ & 93 & $51.67 \%$ & 0.077 \\
\hline Positive & 59 & $39.86 \%$ & 87 & $48.33 \%$ & \\
\hline \multicolumn{6}{|l|}{ PR status } \\
\hline Negative & 56 & $37.84 \%$ & 91 & $56.88 \%$ & 0.001 \\
\hline Positive & 92 & $62.16 \%$ & 69 & $43.13 \%$ & \\
\hline \multicolumn{6}{|l|}{ Her-2 status } \\
\hline Negative & 43 & $29.05 \%$ & 68 & $40.00 \%$ & 0.033 \\
\hline Positive & 105 & $70.94 \%$ & 102 & $60.00 \%$ & \\
\hline
\end{tabular}

\section{Results}

SIRT 3 expression and clinicopathological characteristics of breast cancer patients

Typical SIRT 3 Immunohistochemical stainings are shown in Fig. 1. The immunoreactivities were primarily observed in cytoplasm. We analyzed the associations between the SIRT 3 expression level and the clinicopathological characteristics of patients, including age, histological type, tumor size, lymph node stage, tumor grade, menopausal status, and status of ER, PR, and HER-2 (Table 1). SIRT 3 expression was significantly correlated with lymph node metastasis $(P<0.001)$, tumor grade $(P=0.001)$, tumor size $(\mathrm{P}<0.001)$, Her-2 positive status ( $\mathrm{p}=0.033)$, but unrelated with the patient's age $(\mathrm{P}=0.353)$, menopausal status $(P=0.272)$, histological subtype $(P=0.429)$, and ER status $(P=0.077)$. 

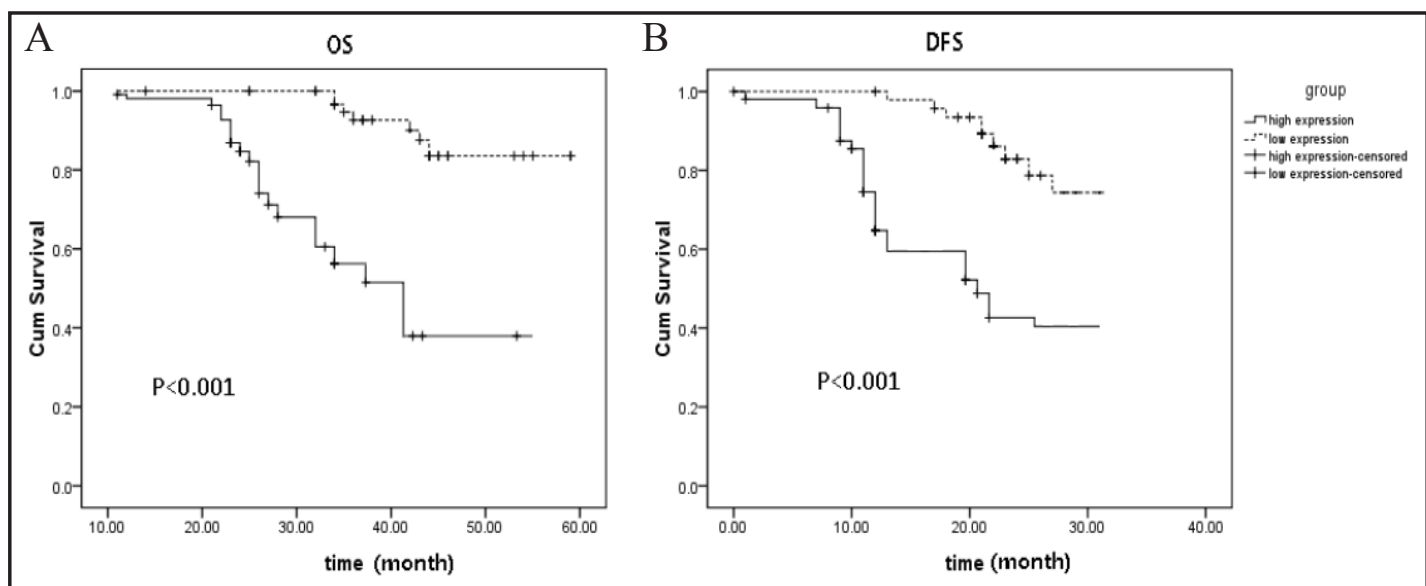

Fig. 2. SIRT 3 expression status with the DFS as well as OS of breast cancer patients were studied by log-rank test. Kaplan-Meier survival curves are shown in Fig. 2a and 2b. Patients with high expression of SIRT 3 had shorter OS and DFS than those with low expression (33.8 \pm 4.5 vs. $45.5 \pm 6.9$, months, $\mathrm{P}<0.001$ and $15.8 \pm 7.2$ vs. $23.9 \pm 5.7$, months, $\mathrm{P}<0.001$ ).

Table 2. Univariate Cox proportional regression analysis on OS and DFS of breast cancer patients

\begin{tabular}{|c|c|c|c|c|c|c|}
\hline \multirow[t]{2}{*}{ Variable } & \multicolumn{3}{|c|}{$O S$} & \multicolumn{3}{|c|}{ DFS } \\
\hline & $H R$ & $95 \% C I$ & $P$ & $H R$ & $95 \% C I$ & $P$ \\
\hline \multicolumn{7}{|l|}{ SIRT3 } \\
\hline Low expression & 1 & & $<0.001$ & 1 & & $<0.001$ \\
\hline High expression & 3.015 & $2.761-4.981$ & & 2.766 & $1.517-4.281$ & \\
\hline \multicolumn{7}{|l|}{ Tumor size } \\
\hline$\leq 2 \mathrm{~cm}$ & 1 & & 0.0016 & 1 & & 0.008 \\
\hline$>2 \mathrm{~cm}$ & 2.622 & $1.692-4.027$ & & 2.853 & $1.825-4.736$ & \\
\hline \multicolumn{7}{|c|}{ Lymph node metastasis } \\
\hline Negative & 1 & & $<0.001$ & 1 & & $<0.001$ \\
\hline positive & 3.078 & $1.698-5.668$ & & 3.305 & $2.376-5.428$ & \\
\hline \multicolumn{7}{|l|}{ Histology Grade } \\
\hline$I$ & 1 & & 0.0014 & 1 & & $<0.001$ \\
\hline II/III & 2.768 & $1.981-4.447$ & & 3.134 & $2.471-5.003$ & \\
\hline
\end{tabular}

Table 3. Multivariate Cox proportional regression analysis on OS and DFS of breast cancer patients

\begin{tabular}{|c|c|c|c|c|c|c|}
\hline \multirow{2}{*}{ Variable } & \multicolumn{3}{|c|}{ OS } & \multicolumn{3}{|c|}{ DFS } \\
\hline & $H R$ & $95 \% C I$ & $P$ & $H R$ & $95 \% C I$ & $P$ \\
\hline SIRT3 & & & & & & \\
\hline Low expression & 1 & & $<0.001$ & 1 & & 0.003 \\
\hline High expression & 2.532 & $1.8251-3.668$ & & 1.825 & $1.253-3.262$ & \\
\hline Histology Grade & & & & & & \\
\hline$I$ & 1 & & 0.0021 & 1 & & 0.009 \\
\hline$I I / I I I$ & 2.224 & $1.254-3.145$ & & 1.831 & $1.378-3.027$ & \\
\hline Lymph node metastasis & & & & & & \\
\hline Negative & 1 & & $<0.001$ & 1 & & $<0.001$ \\
\hline positive & 2.922 & $1.573-4.982$ & & 3.021 & $2.034-5.115$ & \\
\hline
\end{tabular}

High level of SIRT 3 expression indicates a poor prognosis

The relation of SIRT 3 expression status with the DFS as well as OS of breast cancer patients was analyzed. The OS and DFS were $64.3 \%$ and $66.7 \%$ in patients with high SIRT 3 expression level, and 88.9 and $84.8 \%$ in patients with low SIRT 3 expression level, respectively (both $\mathrm{P}<0.05$ ). Patients with high expression of SIRT 3 had shorter OS and DFS than those with low expression (33.8 \pm 4.5 vs. $45.5 \pm 6.9$, months, $\mathrm{P}<0.001$ and $15.8 \pm 7.2$ vs. $23.9 \pm 5.7$, months, $\mathrm{P}<0.001$ ). Kaplan-Meier survival curves are shown in Fig. $2 \mathrm{a}$ and $2 \mathrm{~b}$. 
Fig. 3. SIRT3 mRNA and protein expression in breast cancer cell lines after SIRT 3 siRNA transfection. Both the SIRT3 mRNA (Fig. 3a) and protein expression (Fig. 3b) were significantly decreased in MDAMB-231 and BT-549 cells transfected with SIRT 3 siRNA compared with cells transfected with control siRNA.

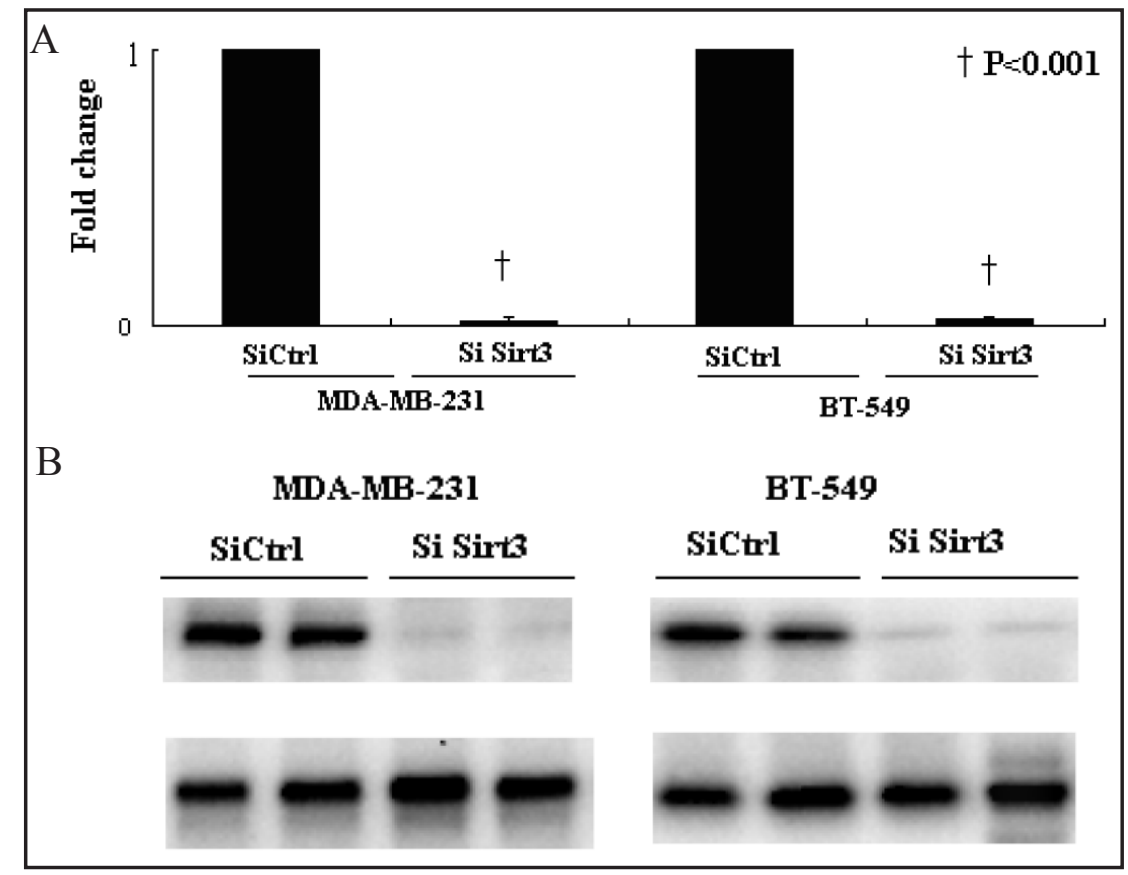

Univariate and multivariate analyses were performed by using Cox proportional hazard model to evaluate the impact of SIRT 3 expression and other pathological factors on the prognosis of breast cancer patients (Table 2). Univariate analysis screened out four statistically significant variables: lymph node metastasis $(\mathrm{P}=0.011)$, tumor size $(\mathrm{P}=0.004)$, tumor grade $(\mathrm{P}=0.004)$, and SIRT 3 expression $(\mathrm{P}<0.001)$. In multivariate analyses, SIRT 3 expression, tumor grade and lymph node metastasis were found to be associated with poor OS and DFS in breast cancer patients (Table 3).

SIRT 3 Regulates Breast Cancer Cells Proliferation, Migration and Invasion

Real-time PCR and Western blot results show significant SIRT 3inhibition at mRNA and protein level in either MDA-MB-231 or BT-549 cells transfected with SIRT 3 siRNA compared with cells transfected with control siRNA (Fig. 3).

The results of MTT cell proliferation assays revealed slowed proliferation rates were significantly inhibited in either MDA-MB-231 or BT-549 breast cancer cells after SIRT 3 siRNA transfection (Fig. 4a and 4b). Cell migration assay showed that SIRT 3 inhibition significantly decreased the cells migration ability of MDA-MB-231 and BT-549 cells by $79 \%$ and $68 \%$, respectively (Fig. 4c and 4d). Furthermore, silencing of SIRT 3 gene by siRNA dramatically inhibited the invasive ability of MDA-MB- 231 and BT- 549 cells by $81 \%$ and $72 \%$, respectively (Fig. 4e and 4f).

\section{Discussion}

In the present study, we investigated the role of SIRT 3 expression in the clinical features and prognosis of breast cancer patients. We found that the SIRT 3 expression was significantly correlated with, lymph node metastasis, higher pathological grade, bigger tumor size. SIRT 3 expression levels are negatively related to DFS as well as OS of breast cancer patients. Univariate and multivariate analyses confirmed that SIRT 3 expression was significantly associated with poor OS and DFS in breast cancer patient. Our in vitro study revealed that the SIRT 3 knockdown by siRNA technique apparently reduced cell proliferation, migration and invasion. Our results suggest that elevated SIRT 3 may serve as a molecular marker for clinical feature and prognosis for breast cancer patients. 


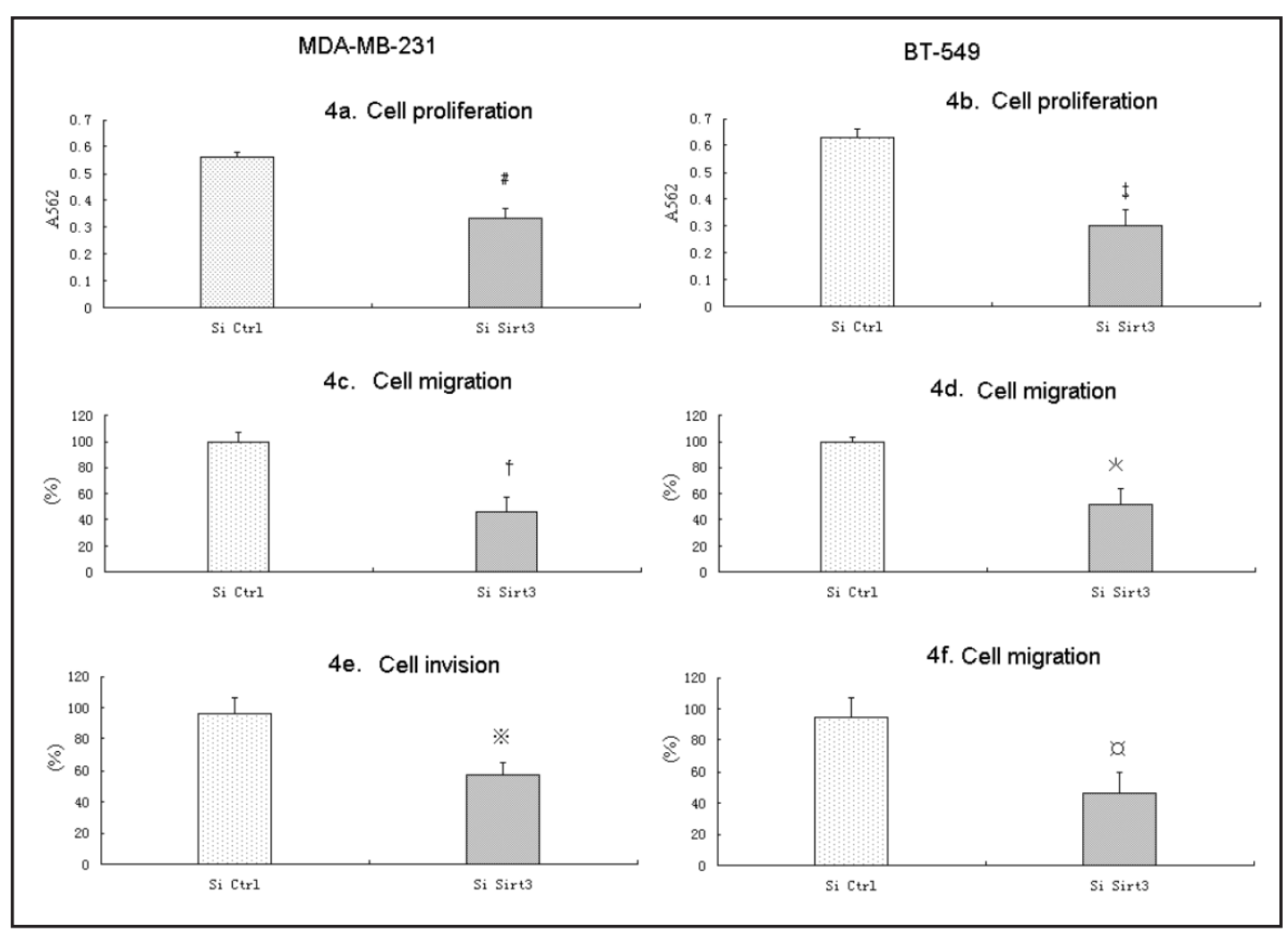

Fig. 4. SIRT 3 regulates breast cancer cells proliferation, migration and invasion. Fig. 4a and 4b show the proliferation rates were significantly inhibited in either MDA-MB-231 or BT-549 breast cancer cells after SIRT 3 siRNA transfection. Fig.. 4c and 4d reveal that SIRT 3 inhibition significantly decreased the cells migration ability of MDA-MB-231 and BT-549 cells by 79\% and 68\%, respectively. Fig. 4e and 4f indicate that silencing of SIRT 3 gene by siRNA dramatically inhibited the invasive ability of MDA-MB-231 and BT-

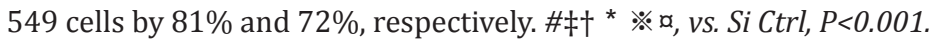

Among the Sirtuin family members, SIRT3 is a mitochondrial protein which is the only member linked to longevity in humans. Increasing evidence suggest that SIRT3 plays an important role in controlling cell proliferative and survival, cancer invasion and metastasis as well as inflammation and the immune response. SIRT3 is suggested as a potential drug target for cancer treatment.

However, there is discrepancy in the literature regarding the role of SIRT3 in cancer. SIRT3 is overexpressed to a greater extent in several human oral cancer cells and tissues than in normal controls, and SIRT3 downregulation in these cells inhibited oral cancer cell growth and proliferation and enhanced radio- and chemo-therapeutic drug cytotoxicity [19]. High expression of SIRT3 is associated with a shorter survival time in esophageal cancer patients[20, 21]. p53-induced growth arrest in human bladder cancer is regulated by the mitochondrial SIRT3 deacetylase [22, 23]. All these observations suggest a tumor promoter effect of SIRT3. In contrast, many others reported role of SIRT3 as a tumor suppressor protein in several type of cancers. Down-regulation of sirtuin 3 is associated with poor prognosis in hepatocellular carcinoma after resection [24-26]. The expression level of SIRT3 was inversely correlated with clinicopathological variable, including tumor infiltration, tumor differentiation and tumor stage and 5-year survival of patients with gastric cancer [27].

The role of SIRT3 in breast cancer is also controversial. Ashraf et al. reported no overall difference observed in SIRT3 expression between breast cancer and normal breast tissue. However, on subdividing the cancer biopsies by nodal status, SIRT3 expression was dramatically over-expressed in node-positive breast cancer compared to normal breast tissue $[28,29]$. In a very recent study, Desouki et al. found that SIRT3 protein expression is 
significantly lower in breast cancer tissue compared with normal breast epithelium. Patients with breast cancer in the lower SIRT3 expression had a significantly shorter relapse-free survival [30]. In the present study, we found that the SIRT 3 expression was significantly correlated with, lymph node metastasis, higher pathological grade, bigger tumor size $(>2 \mathrm{~cm})$, higher percentage of Her-2 positive status. It seems that our finding is consistent with that of Ashraf et al., but against that of Desouki et al.. We think the difference in harvesting sample, tumor tissue blood and oxygen supply might be reason for this discrepancy.

In the prognosis analyses of this study, we reported a close relation of SIRT 3 expression status and the DFS as well as OS of breast cancer patients. High expression of SIRT 3 was found as a detrimental factor for prognosis since patients with high SIRT 3expression had shorter OS and DFS than those with low expression. Univariate and multivariate COX analyses confirmed that SIRT 3 expression was significantly associated with poor OS and DFS in breast cancer patient.

SIRT3 promotes cell proliferation and survival in oral cancer carcinogenesis. In addition, under genotoxic stress, mitochondrial SIRT3 and SIRT4 were required to protect against genotoxic cell death in human embryonic kidney (HEK293) and fibrosarcoma cell lines [31]. SIRT3 augments Ku70-Bax interactions, prevents Bax translocation to the mitochondria, and prevents apoptosis during stress-mediated conditions [32]. In our study, we inhibited the SIRT3 by Si RNA technique. We found that the proliferation rate, migration and invasiveness rate were all significantly reduced after Si SIRT3 transfection in two cultured breast cell lines. This in vitro study provided evidence the way SIRT 3 influence the biological behavior of breast cancer cells. This observation was consistent with the above-mentioned results.

The role of ROS in cancer cell biology is well established. SIRT3 is a major regulator of ROS level in cells. The intact SIRT3 level in cancer cells can maintain the cellular ROS levels at the appropriate level for maintaining a proliferative and aggressive phenotype. Collectively, SIRT 3 is essential to maintain breast cancer cell viability and migration and invasive ability, thus contribute to poorer prognosis in patients.

In summary, we demonstrated that SIRT 3 plays an important role in human breast cancer pathogenesis. Increased SIRT 3 expression may facilitate tumor progression by enhancing cell growth, migration and invasion. Our results imply that SIRT 3 may serve as a prognostic marker as well as a potential therapeutic target for breast cancer.

\section{References}

1 Johnson-Thompson MC, Guthrie J: Ongoing research to identify environmental risk factors in breast carcinoma. Cancer 2000;88:1224-1229.

-2 Trentham-Dietz A, Newcomb PA, Storer BE, Remington PL: Risk factors for carcinoma in situ of the breast. Cancer Epidemiol Biomarkers Prev 2000;9:697-703.

-3 Chen L, Fan Y, Lang RG, Guo XJ, Sun YL, Fu L: [diagnosis and prognosis study of breast carcinoma with micropapillary component]. Zhonghua Bing Li Xue Za Zhi 2007;36:228-232.

-4 Cao AY, He M, Liu ZB, Di GH, Wu J, Lu JS, Liu GY, Shen ZZ, Shao ZM: Outcome of pure mucinous breast carcinoma compared to infiltrating ductal carcinoma: A population-based study from china. Ann Surg Oncol 2012;19:3019-3027.

>5 Fan L, Zheng Y, Yu KD, Liu GY, Wu J, Lu JS, Shen KW, Shen ZZ, Shao ZM: Breast cancer in a transitional society over 18 years: Trends and present status in shanghai, china. Breast Cancer Res Treat 2009;117:409-416.

-6 Engel J, Holzel D: [risk and prognosis of corpus carcinomas after tamoxifen treatment of breast carcinoma]. Strahlenther Onkol 2001;177:371.

7 Sack H: [risk factors for local recurrence and distant metastases after breast conserving therapy of ductal carcinoma in situ]. Strahlenther Onkol 2001;177:557.

8 Varadarajan R, Edge SB, Yu J, Watroba N, Janarthanan BR: Prognosis of occult breast carcinoma presenting as isolated axillary nodal metastasis. Oncology 2006;71:456-459.

-9 Takeuchi H, Tsuji K, Ueo H, Kano T, Maehara Y: Clinicopathological feature and long-term prognosis of apocrine carcinoma of the breast in japanese women. Breast Cancer Res Treat 2004;88:49-54. 
He et al.: SIRT 3 Expression in Breast Cancer Patients

10 Hanrahan EO, Valero V, Gonzalez-Angulo AM, Hortobagyi GN: Prognosis and management of patients with node-negative invasive breast carcinoma that is $1 \mathrm{~cm}$ or smaller in size (stage 1 ; $\mathrm{t} 1 \mathrm{a}, \mathrm{bn} 0 \mathrm{~m} 0$ ): A review of the literature. J Clin Oncol 2006;24:2113-2122.

11 Sauve AA, Wolberger C, Schramm VL, Boeke JD: The biochemistry of sirtuins. Annu Rev Biochem 2006;75:435-465.

12 Zeng H, He X, Hou X, Li L, Chen JX: Apelin gene therapy increases myocardial vascular density and ameliorates diabetic cardiomyopathy via upregulation of sirtuin 3. Am J Physiol Heart Circ Physiol 2014;306:H585-597.

13 Holloway KR, Barbieri A, Malyarchuk S, Saxena M, Nedeljkovic-Kurepa A, Cameron Mehl M, Wang A, Gu X, Pruitt K: Sirt1 positively regulates breast cancer associated human aromatase (cyp19a1) expression. Mol Endocrinol 2013;27:480-490.

14 Saxena M, Dykes SS, Malyarchuk S, Wang AE, Cardelli JA, Pruitt K: The sirtuins promote dishevelled-1 scaffolding of tiam1, rac activation and cell migration. Oncogene 2013

15 Holloway KR, Calhoun TN, Saxena M, Metoyer CF, Kandler EF, Rivera CA, Pruitt K: Sirt1 regulates dishevelled proteins and promotes transient and constitutive wnt signaling. Proc Natl Acad Sci U S A 2010;107:9216-9221.

16 Moore RL, Faller DV: Sirt1 represses estrogen-signaling, ligand-independent eralpha-mediated transcription, and cell proliferation in estrogen-responsive breast cells. J Endocrinol 2013;216:273-285.

17 Elangovan S, Ramachandran S, Venkatesan N, Ananth S, Gnana-Prakasam JP, Martin PM, Browning DD, Schoenlein PV, Prasad PD, Ganapathy V, Thangaraju M: Sirt1 is essential for oncogenic signaling by estrogen/estrogen receptor alpha in breast cancer. Cancer Res 2011;71:6654-6664.

18 Remmele W, Stegner HE: [recommendation for uniform definition of an immunoreactive score (irs) for immunohistochemical estrogen receptor detection (er-ica) in breast cancer tissue]. Pathologe 1987;8:138140.

19 Alhazzazi TY, Kamarajan P, Joo N, Huang JY, Verdin E, D'Silva NJ, Kapila YL: Sirtuin-3 (sirt3), a novel potential therapeutic target for oral cancer. Cancer 2011;117:1670-1678.

20 Zhao Y, Yang H, Wang X, Zhang R, Wang C, Guo Z: Sirtuin-3 (sirt3) expression is associated with overall survival in esophageal cancer. Ann Diagn Pathol 2013;17:483-485.

21 Hou X, Hu Z, Xu H, Xu J, Zhang S, Zhong Y, He X, Wang N: Advanced glycation endproducts trigger autophagy in cadiomyocyte via rage/pi3k/akt/mtor pathway. Cardiovasc Diabetol 2014;13:78.

22 Li S, Banck M, Mujtaba S, Zhou MM, Sugrue MM, Walsh MJ: P53-induced growth arrest is regulated by the mitochondrial sirt3 deacetylase. PLoS One 2010;5:e10486.

23 Yi L, Hou X, Zhou J, Xu L, Ouyang Q, Liang H, Zheng Z, Chen H, Xu M: Hif-1alpha genetic variants and protein expression confer the susceptibility and prognosis of gliomas. Neuromolecular Med 2014

-24 Wang JX, Yi Y, Li YW, Cai XY, He HW, Ni XC, Zhou J, Cheng YF, Jin JJ, Fan J, Qiu SJ: Down-regulation of sirtuin 3 is associated with poor prognosis in hepatocellular carcinoma after resection. BMC Cancer 2014;14:297.

25 Li L, Zeng H, Hou X, He X, Chen JX: Myocardial injection of apelin-overexpressing bone marrow cells improves cardiac repair via upregulation of sirt3 after myocardial infarction. PLoS One 2013;8:e71041.

26 Hou X, Hu Z, Huang X, Chen Y, He X, Xu H, Wang N: Serum osteopontin, but not opn gene polymorphism, is associated with lvh in essential hypertensive patients. J Mol Med (Berl) 2014;92:487-495.

27 Yang B, Fu X, Shao L, Ding Y, Zeng D: Aberrant expression of sirt3 is conversely correlated with the progression and prognosis of human gastric cancer. Biochem Biophys Res Commun 2014;443:156-160.

-28 Ashraf N, Zino S, Macintyre A, Kingsmore D, Payne AP, George WD, Shiels PG: Altered sirtuin expression is associated with node-positive breast cancer. Br J Cancer 2006;95:1056-1061.

29 Hou XW, Son J, Wang Y, Ru YX, Lian Q Majiti W, Amazouzi A, Zhou YL, Wang PX, Han ZC: Granulocyte colonystimulating factor reduces cardiomyocyte apoptosis and improves cardiac function in adriamycin-induced cardiomyopathy in rats. Cardiovasc Drugs Ther 2006;20:85-91.

-30 Desouki MM, Doubinskaia I, Gius D, Abdulkadir SA: Decreased mitochondrial sirt3 expression is a potential molecular biomarker associated with poor outcome in breast cancer. Hum Pathol 2014;45:1071-1077.

-31 Yang H, Yang T, Baur JA, Perez E, Matsui T, Carmona JJ, Lamming DW, Souza-Pinto NC, Bohr VA, Rosenzweig A, de Cabo R, Sauve AA, Sinclair DA: Nutrient-sensitive mitochondrial nad+ levels dictate cell survival. Cell 2007;130:1095-1107.

-32 Alhazzazi TY, Kamarajan P, Verdin E, Kapila YL: Sirt3 and cancer: Tumor promoter or suppressor? Biochim Biophys Acta 2011;1816:80-88. 